\title{
Master integrals for all unitarity cuts of massless four-loop propagators
}

\author{
Vitaly Magerya* \\ II. Institut für Theoretische Physik, Universität Hamburg, \\ Luruper Chaussee 149, 22761 Hamburg, Germany \\ E-mail: vitaly.mageryaddesy.de
}

\section{Andrey Pikelner}

Bogoliubov Laboratory of Theoretical Physics, Joint Institute for Nuclear Research, Joliot-Curie 6, 141980 Dubna, Russia

E-mail: pikelneretheor.jinr.ru

\begin{abstract}
Among the unitarity cuts of massless 4-loop propagators two classes have remained unknown until recently: 2-loop 3-particle cuts, and 1-loop 4-particle cuts. In this article we shall discuss the calculation that completes the master integrals for these cuts: both the motivation and the methods (including dimensional recurrence relations and direct integration at higher space-time dimensions).
\end{abstract}

14th International Symposium on Radiative Corrections (RADCOR2019)

9-13 September 2019

Palais des Papes, Avignon, France

\footnotetext{
${ }^{*}$ Speaker.
} 


\section{Introduction}

Continuing the effort started last year in [1], we have finished the calculation of all master integrals for unitarity cuts of massless 4-loop propagators [2]. Here we would like to briefly explain the motivation behind, and the method of calculation of these integrals.

Motivation: splitting functions. The main motivation for our work are the NNLO corrections to time-like splitting functions. As a quick recap: the time-like splitting functions $P_{a b}^{T}(x)$ (for $\left.a, b \in\left\{q_{i}, \bar{q}_{i}, g\right\}\right)$ act as the kernels in the DGLAP-like scale evolution equations for the parton fragmentation distributions, and are related to the space-like splitting functions $P_{a b}^{S}(x)$ that play the same role for the parton distribution functions. The time-like splitting functions are currently known in the leading color limit up to $\mathrm{N}^{3} \mathrm{LO}$ [3]; in full they are known only up to NNLO [4, 5, 6], with a caveat: some of the NNLO terms of $P_{q g}^{T}$ and $P_{g q}^{T}$ are still undetermined. The reason for this caveat is that the time-like case was calculated via an analytic continuation from the space-like case. At the leading order these two are related simply by $P_{a b}^{T}(x)=-x P_{a b}^{S}(1 / x)$, but at higher orders this relation does not hold anymore, with the reason being that, roughly speaking, terms like $\ln (1-1 / x)$ that appear in $P_{a b}^{S}(1 / x)$ starting at NLO may need to be continued either as $\ln (1 / x-1)+i \pi$ or as $\ln (1 / x-1)-i \pi$ depending on the term, which creates an uncertainty, and makes it impossible to unambiguously restore the full $P_{a b}^{T}$ from just $P_{a b}^{S}$. To overcome this uncertainty, a direct calculation of $P_{a b}^{T}(x)$ is required.

At NNLO the "direct" calculation of $P_{g q}^{T}$ can be performed [7] by first calculating the differential cross-section $d \sigma_{p} / d x$ of

$$
e^{+} e^{-} \rightarrow \gamma^{*} \rightarrow \text { parton } p+\ldots,
$$

in the energy fraction of one of the outgoing partons, $x=2 q \cdot k_{p} / q^{2}$, and then the splitting functions can be read off from the poles of this cross-section,

$$
\frac{d \sigma_{p}}{d x} \sim \cdots+\left(\frac{\alpha_{s}}{2 \pi}\right)\left\{\cdots-\frac{1}{\varepsilon} P_{p q}^{(0) T}\right\}+\left(\frac{\alpha_{s}}{2 \pi}\right)^{2}\left\{\cdots-\frac{1}{2 \varepsilon} P_{p q}^{(1) T}\right\}+\left(\frac{\alpha_{s}}{2 \pi}\right)^{3}\left\{\cdots-\frac{1}{3 \varepsilon} P_{p q}^{(2) T}\right\},
$$

where $P_{a b}^{(i) T}(x)$ are the terms of $\alpha_{s}$ expansion of $P_{a b}^{T}$. Note that the needed NNLO correction $P_{g q}^{(2) T}(x)$ shows up at $\alpha_{s}^{3}$ of $d \sigma_{g} / d x$, so an $\mathrm{N}^{3} \mathrm{LO}$ calculation is needed.

To obtain $P_{q g}^{(2) T}$ the calculation is the same, except that in stead of a $\gamma^{*}$, a particle that couples to gluons is needed: for example a scalar Higgs in an effective theory with a Higgs-gluon coupling. Splitting functions are process-independent, so there is some leeway here.

Of course completing this calculation requires the knowledge the master integrals for $d \sigma / d x$ at $\mathrm{N}^{3} \mathrm{LO}$, and these are not currently known. With some preliminary work already done in this direction we think that the total number of these master integrals is around 500, and that we can calculate them using the differential equations method (via the $\varepsilon$-forms constructed by Fuchsia [8]), as long as there would be a way to fix the integration constants that arise in the solution. Following [9] we propose to do this by exploiting the observation that an integral over all $x$ values should turn a differential cross-section into a fully inclusive one, i.e. $\int d x \frac{d \sigma}{d x}(x)=\sigma$. Applying this observation to each differential master integral, we can fix its integration constants as long as the corresponding fully inclusive integral is known. This brings us to the topic at hand: unitarity cuts of propagators are exactly the master integrals for the required fully inclusive cross-sections. Let us illustrate this point. 
Cut integrals. For a fully inclusive decay cross-section of an off-shell particle of momenta $q$ into $n$ partons of momenta $p_{i}$, the cross-section is given by

$$
\sigma \sim \sum_{n} \int d \mathrm{PS}_{n}\left|\left\langle p_{1}, \ldots, p_{n}|S| q\right\rangle\right|^{2}=\sum_{n} \int d \mathrm{PS}_{n} \mid
$$

where $d \mathrm{PS}_{n}$ is the $n$-particle phase-space element,

$$
d \mathrm{PS}_{n}=(2 \pi)^{d} \delta^{d}\left(p_{1}+\cdots+p_{n}-q\right) \prod_{i=1}^{n} \frac{d^{d} p_{i}}{(2 \pi)^{d}} 2 \pi \delta\left(p_{i}^{2}\right) \Theta\left(p_{i}^{0}\right),
$$

and the graphs stand for Feynman integrals. Expanding the module squared, each term gives a cut propagator, denoted the following way:

$$
\int d \mathrm{PS}_{3} \mathrm{C}_{2}\left(\mathrm{PS}_{3}\right.
$$

For $\mathrm{N}^{3} \mathrm{LO}\left(\alpha_{s}^{3}\right)$ we need all sets of cuts of 4-loop propagators: 2-particle cuts, 3-particle, 4particle, and 5-particle cuts. Among these, the 2-particle cuts correspond to 3-loop form-factors, and have been completed around 2010 [10,11, 12]; the 5-particle cuts are purely phase-space integrals and where calculated last year [1]. Some subset of for 3- and 4-particle cuts have also been calculated in 2015 [13], but the majority where unknown until the full set of the master integrals was completed in [2].

Identifying the master integrals. To calculate the cut master integrals we first need to identify them. This is particularly easy if one starts with the master integrals for the propagators themselves (without cuts): there are 28 such master integrals, and their values are known from [14, 15].

To identify the cut master integrals, first, for each of the 28 propagator master integrals one needs to construct all of its possible cuts. For example:

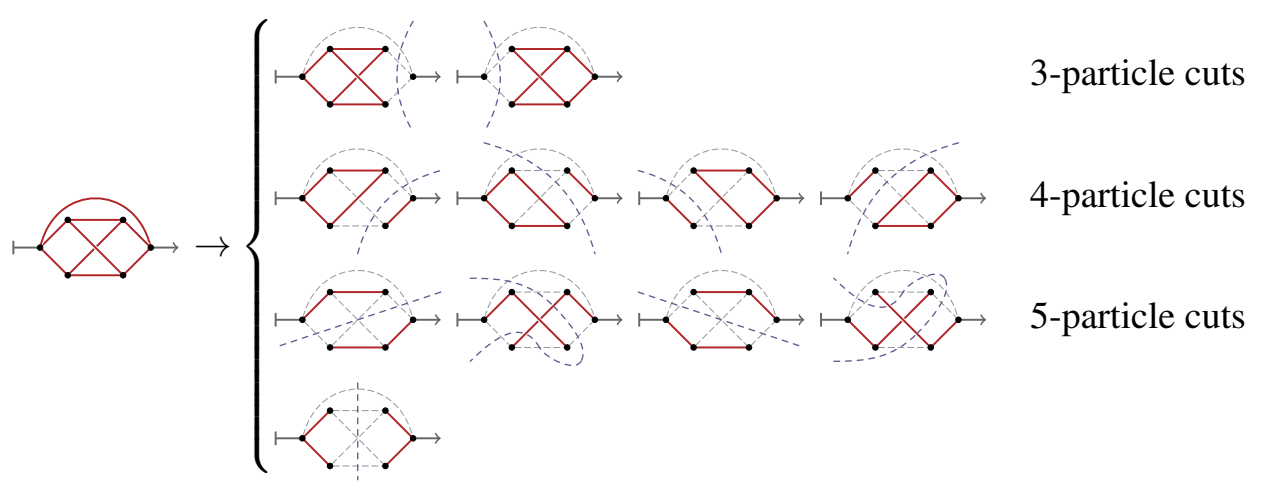

Second, from the resulting set of cuts one needs to remove all the symmetric duplicate integrals. For example, all of the following four integrals are identical up to a complex conjugation:

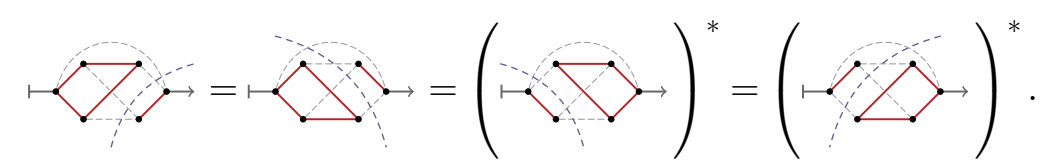

And this is all, the resulting set will be the final answer. There are neither additional IBP relations between the remaining integrals, nor are there any additional master integrals. 
Proceeding this way we have identified the set of master integrals for 4-particle cuts: 35 integrals in total, depicted in Table 2; and the same for 3-particle cuts: 27 integrals in total, depicted in Table 3.

\section{Four-particle cut masters via dimensional recurrence relations}
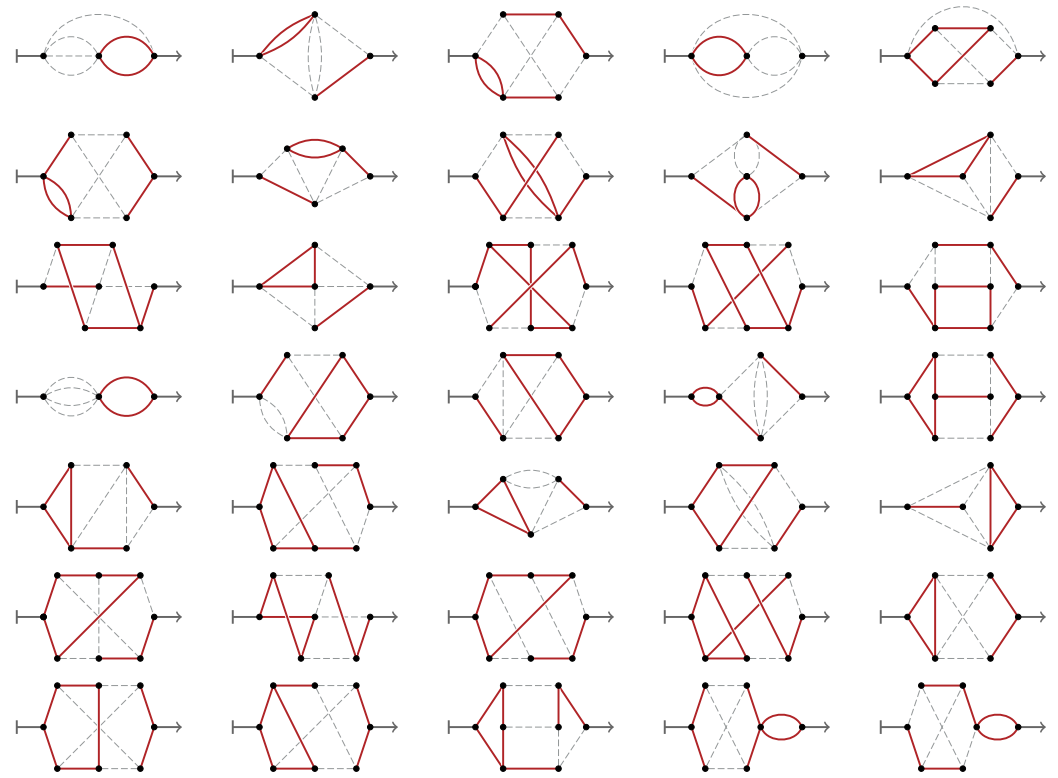

Figure 1: All 35 master integrals for 4-particle cuts of 4-loop propagators.

Direct analytic integration? Four-particle cut integrals consist of a 4-particle phase space and a 1-loop amplitude. Calculating these can be as easy as inserting the values of the 1-loop amplitudes (which are known for arbitrary space-time dimension $d$ in terms of hypergeometric functions), and directly integrating them over the 4-particle phase-space. For example knowing the value of a triangle amplitude with all off-shell legs, one might be able to integrate directly

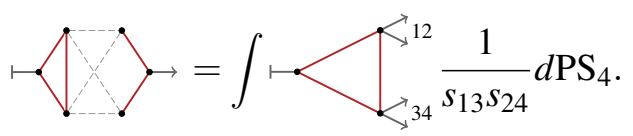

The complication is that 4-particle phase-space has 5 degrees of freedom and a nontrivial shape. Its parametrisation in terms of the scalar products $s_{i j}=\left(p_{i}+p_{j}\right)^{2} / q^{2}$ looks like

$$
d \mathrm{PS}_{4}=\left(q^{2}\right)^{\frac{3 d-4}{2}} \frac{2^{4-4 d} \pi^{\frac{1}{2}-\frac{3}{2} d}}{\Gamma(d-3) \Gamma\left(\frac{d-1}{2}\right)}\left(\Delta_{4}\right)^{\frac{d-5}{2}} \Theta\left(\Delta_{4}\right) \Theta\left(s_{i j}\right) \delta\left(1-\sum s_{i j}\right) \prod d s_{i j}
$$

where $\Delta_{4}$ is the Gram determinant,

$$
\Delta_{4}=\operatorname{det}\left|\begin{array}{cccc}
0 & s_{12} & s_{13} & s_{14} \\
s_{12} & 0 & s_{23} & s_{24} \\
s_{13} & s_{23} & 0 & s_{34} \\
s_{14} & s_{24} & s_{34} & 0
\end{array}\right|,
$$


and the shape of the integration region is given by $\Theta\left(\Delta_{4}\right) \Theta\left(s_{i j}\right)$. This is quite a non-trivial shape! Parametrisations exist that simplify this shape to a hypercube, e.g. the "tripole parametrisation" [16] given by the following change of variables:

$$
\begin{aligned}
& s_{12} \rightarrow(1-t)(1-y)(1-z), \\
& s_{13} \rightarrow y(1-t-v+t v+t v z-2(1-2 \xi) \sqrt{(1-t)(1-v) t v z}), \\
& s_{14} \rightarrow v y(1-z), \\
& s_{23} \rightarrow z(1-y), \\
& s_{24} \rightarrow t(1-y)(1-z), \\
& s_{34} \rightarrow y(t-t v+v z-t v z+2(1-2 \xi) \sqrt{(1-t)(1-v) t v z}),
\end{aligned}
$$

but such parametrisations contain square roots, making analytic integration only possible in the simple cases when the integrand turns out to be free from these roots. This is not the case for the majority of the master integrals. For this reason we turn to a different approach: solving dimensional recurrence relations.

Dimensional recurrence relations. Using a parametric representation of integrals (Feynman for uncut, Baikov-like for cut) and the IBP tables, we can obtain dimensional recurrence relations [17] (DRR) relating the set master integrals at a raised dimension $I_{i}(d+2)$ to the initial integrals:

$$
I_{i}(d+2)=M_{i j}(d) I_{j}(d) .
$$

These relations can be solved, and for the 4-particle cuts specifically, two features of $M_{i j}$ simplify this solution:

- The DRR matrix $M_{i j}$ is triangular (for $j>i M_{i j}=0$ ). This means that each $I_{i}$ can be solved for one by one, with only the previous $I_{j<i}$ required for the solution—no coupled blocks need to be considered. This structure is not surprising, seeing that at most one master integral is present in each IBP sector for the propagators; the cuts retain this structure as well.

- The diagonal of $M$ is factorizable, with factors linear in $d: M_{i i}=C \prod_{k}\left(d / 2-a_{k}\right)^{n_{k}}$.

The general solution to DRR has the form of

$$
I_{i}(d)=H_{i}(d) \omega_{i}(d)+R_{i}(d)
$$

where

- $H_{i}$ is a homogeneous solution, $H_{i}(d+2)=M_{i i}(d) H_{i}(d)$. For a factorizable diagonal with no coupled blocks this can immediately be constructed as

$$
H_{i}(d)=C^{\frac{d}{2}} \prod_{k}\left\{\Gamma^{n_{k}}\left(\frac{d}{2}-a_{k}\right) \quad \text { or } \quad(-1)^{\frac{d}{2} n_{k}} \Gamma^{-n_{k}}\left(a_{k}-\frac{d}{2}+1\right)\right\},
$$

where both forms of the factors are acceptable, and generally one chooses one or the other depending on where it would be most convenient to have the poles and zeros of $H(d)$ reside: the first form produces poles (or zeros) at $d=2 a_{k}, 2 a_{k}-2,2 a_{k}-4$, etc., while for the second they are at $2 a_{k}-2,2 a_{k}, 2 a_{k}+2$, etc. 
- $R_{i}$ is a particular solution. In the absence of coupled blocks it can be constructed as an infinite sum,

$$
R_{i}(d)=H_{i}(d)\left\{-\sum_{k=0}^{\infty} \quad \text { or } \quad \sum_{k=-\infty}^{-1}\right\} H_{i}^{-1}(d+2 k+2) \sum_{j<i} M_{i j}(d+2 k) J_{j}(d+2 k),
$$

where the direction of summation is chosen based on which one converges. The appealing feature of this sum is that it converges geometrically, and thus can be evaluated numerically with thousands of digits of precision (as a series in $\varepsilon$ ); its analytic form in terms of multiple zeta values [18] (MZVs) can then be restored using the PSLQ algorithm [19].

- $\omega$ is an arbitrary periodic function, $\omega(d+2)=\omega(d)$. This function can not be determined from DRR alone; it plays the same role in the solution of DRR as the integration constants play in the solution of differential equations.

To find $\omega(d)$ is the main challenge of the method. Here is an outline of our method (following [20]):

1. Find as stripe of width 2 for $d$, such that $I_{i}(d)$ is finite if $\operatorname{Re}(d) \in\left(d_{0}, d_{0}+2\right]$, or at least has as few poles as possible. 4-particle cut master integrals $I_{i}$ have surface divergences of UV nature at even $d$, but these can be cancelled by factoring out a 1-loop bubble, so in stead of $I_{i}$ we choose to work with $J_{i} \equiv I_{i} / 1 \bigcirc$, which have no UV poles at all, and no IR poles at $d \geq 6$. Thus, we choose the stripe to be $(6,8]$, where $J_{i}$ are finite.

2. Construct the homogeneous solutions $H_{i}(d)$ with eq. (2.12), and determine their pole locations. In our case these can all be chosen to be finite.

3. Construct the particular solutions $R_{i}(d)$ with eq. (2.13), and find their pole locations by evaluating them numerically for many values of $d$. We use DREAM [21] for this evaluation, and all $R_{i}(d)$ appear to be finite.

4. Analyze the behaviour of $\omega_{i}(d)$ at $\operatorname{Im} d \rightarrow \pm \infty$ by analyzing the same for $J_{i}, H_{i}$, and $R_{i}$. If it can be proven that $\left|\omega_{i}(d)\right| \leq K|\operatorname{Im} d|^{\alpha}$ for some $K$ and $\alpha$, then $\omega_{i}(d)$ can only have this form: $C_{0}+\sum C_{k} \cot \left(\frac{\pi}{2}\left(d-d_{k}\right)\right)$, where $C_{k}$ are some constants, and $d_{k}$ are the location of poles of $\omega_{i}$ (a subset of poles of $J_{i}, H_{i}$ and $R_{i}$ ). This is why it is important to choose a stripe for $d$ where as few poles appear as possible: the more poles, the more constants need to be fixed.

For smooth $J_{i}, H_{i}$, and $R_{i}$ only one choice is possible: a constant, $C_{0}$.

5. Fix the constants in the ansatz from various considerations.

Only one constant is needed for us, and we can determine it from the leading pole of $I_{i}$ at $d$ where it diverges logarithmically in the UV. This pole can be calculated by inserting a mass into the loop and looking at the large mass expansion of the result. Interestingly, we find that for most integrals (except the trivial ones) the constant $C_{0}$ (and thus $\omega_{i}$ ) is zero; a similar situation was already observed previously in [1] for the case of the 5-particle cuts.

Once $\omega_{i}$ are fixed, the masters can be evaluated with DREAM or SUMMERTIME [22] numerically to arbitrary precision, and then restored in terms of MZVs via PSLQ. Here is an example 
result:

$$
\begin{aligned}
\vee & =\frac{(\mapsto)^{*}}{\left(q^{2}\right)^{3}}\left[-6 \zeta_{2} \frac{1}{\varepsilon^{2}}+\left(59 \zeta_{2}-60 \zeta_{3}\right) \frac{1}{\varepsilon}+\left(-203 \zeta_{2}+590 \zeta_{3}-\right.\right. \\
& \left.\left.-129 \zeta_{2}^{2}\right)+\left(288 \zeta_{2}-2030 \zeta_{3}+\frac{2537}{2} \zeta_{2}^{2}+192 \zeta_{2} \zeta_{3}-1806 \zeta_{5}\right) \varepsilon+\mathscr{O}\left(\varepsilon^{2}\right)\right]
\end{aligned}
$$

Overall, we've restored the series up to MZVs of weight 12 . They have poles up to $1 / \varepsilon^{5}$, and zetas up to weight 6 in the $\varepsilon$-finite part.

To cross-check these results we have evaluated them numerically via simple Monte-Carlo (without e.g. sector decomposition) by combining Feynman parametrisation of the 1-loop amplitude with the tripole parametrisation of $d \mathrm{PS}_{4}$. This is possible without running into divergences because UV divergence only comes from the pre-factors of the Feynman parametrisation (which can be factorized), and evaluating the integrals in $d=6-2 \varepsilon$ removes the IR divergences, so the result is finite.

\section{Three-particle cut masters via integration in 6 dimensions}

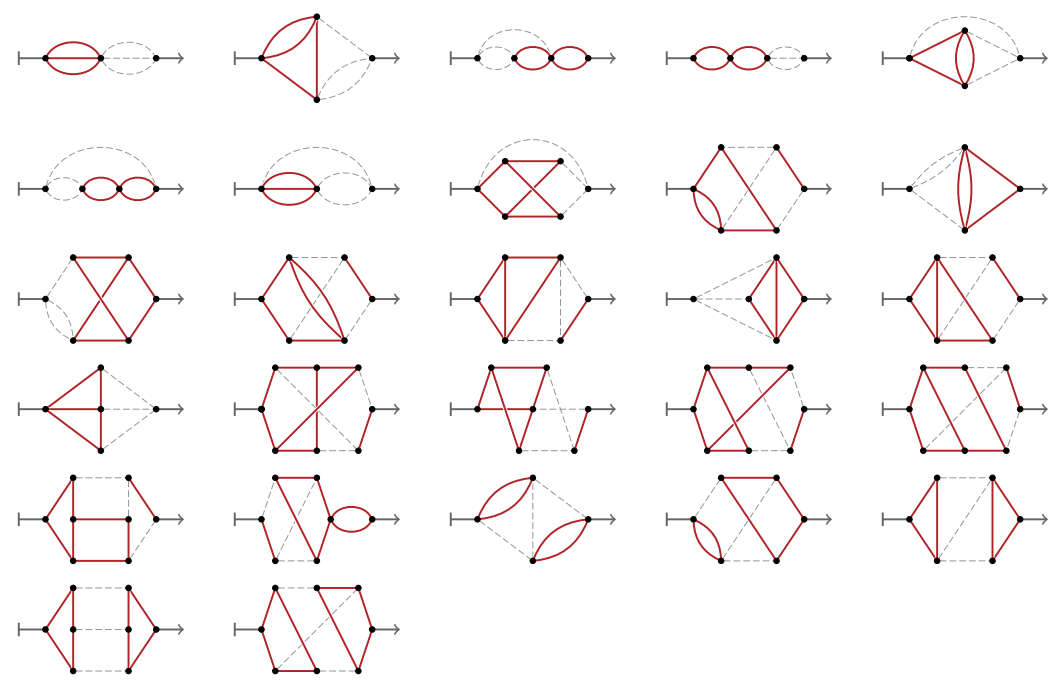

Figure 2: All 27 master integrals for 3-particle cuts of 4-loop propagators.

Solving DRR for 3-particle cuts is much more difficult than for the 4-particle ones because they contain 2-loop amplitudes, and thus many more UV poles from subdivergences, so the ansätze for $\omega_{i}$ may have dozens of free parameters, and we don't have enough information to fix them (yet).

On the flip side, the 3-particle phase space is simpler that the 4-particle one, and we are fully able to represent these master integrals as a 2-loop $1 \rightarrow 3$ amplitude and 3-particle phase space, e.g.

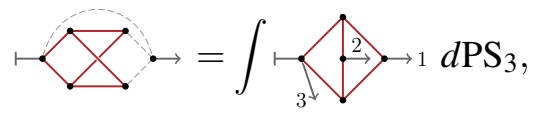


parameterize the phase-space via $s_{i j}=\left(p_{i}+p_{j}\right)^{2} / q^{2}$ as

$$
d \mathrm{PS}_{3}=\left(q^{2}\right)^{d-3} \frac{2^{4-3 d} \pi^{\frac{3}{2}-d}}{\Gamma\left(\frac{d-2}{2}\right) \Gamma\left(\frac{d-1}{2}\right)}\left(s_{12} s_{13} s_{23}\right)^{\frac{d-4}{2}} \delta\left(1-\sum s_{i j}\right) \prod d s_{i j},
$$

and integrate the result — as long as we know the values of the 2-loop $1 \rightarrow 3$ amplitudes — and we do: the master integrals are known from [23, 24] as series in $\varepsilon$ with coefficients being multiple polylogarithms [25] (depending on $s_{12}$ and $s_{13}$ ) up to transcendental weight 4.

There are two caveats here. The first one is that just taking a 2-loop $1 \rightarrow 3$ amplitudes as series in $\varepsilon$, multiplying it by the expansion of $d \mathrm{PS}_{3}$, and integrating order by order in $\varepsilon$ will give infinity in each order:

$$
\int \longmapsto \stackrel{2}{\stackrel{2}{\Rightarrow} \rightarrow 1} d \mathrm{PS}_{3}=\int \frac{C}{s_{12}^{2}}\left(\frac{1}{\varepsilon^{4}}-\frac{2 \ln s_{12}}{\varepsilon^{3}}+\ldots\right) d \mathrm{PS}_{3}=\frac{\infty}{\varepsilon^{4}}+\frac{\infty}{\varepsilon^{3}}+\ldots
$$

The reason is that the integrals are IR-divergent, and for them taking a series in $\varepsilon$ does not commute with integration, so the integral must be taken before the expansion into series.

The solution is to switch to an IR-finite basis of integrals. Any such basis should work, but a simple choice is to take the same list of master integrals only evaluated at $d=6-2 \varepsilon$. This is a dimension high enough to cancel the IR poles, so the integration can be done order by order in $\varepsilon$.

The second caveat is that because the $\varepsilon$-finite part of the propagator master integrals contain MZVs up to weight 7, and the same for 4-particle cuts go up to weight 6 (as we've already seen), it means that we need to know the 2-loop $1 \rightarrow 3$ amplitudes up to at least weight 6 too-but the results in $[23,24]$ only go to weight 4 . For this reason we have recomputed the $1 \rightarrow 3$ master integrals up to weight 7, by:

- writing down differential equation systems for them in $s_{12}$ and $s_{23}$;

- finding a change of basis that simultaneously reduces both systems into an $\varepsilon$-form [26, 27] using Fuchsia ${ }^{1}[8]$

- constructing the general solution, containing a mixture of multiple polylogarithms in the form of $\sum R\left(s_{i j}, C\right) G\left(\left\{0,1,1-s_{13},-s_{13}\right\} ; s_{12}\right) G\left(\{0,1\} ; s_{13}\right)$, where $R$ are some rational functions, $C$ are the integration constants, and $G$ are the multiple polylogarithms;

- fixing the integration constants $C$ by matching with the known single-scale integrals, enforcing regularity at $s_{i j} \rightarrow 1$ (because the integrals are massless), and enforcing regularity at $s_{i j} \rightarrow 0$ for planar integrals if $i$ and $j$ are not adjacent.

With the 2-loop $1 \rightarrow 3$ amplitudes obtained this way up to weight 7 , we can proceed to evaluate the 3-particle cut integrals to the same weight. For this:

1. Use dimensional recurrence for the $1 \rightarrow 3$ amplitudes to get them as series around $d=6-2 \varepsilon$.

2. Multiply that by the $\varepsilon$-expansion of $d \mathrm{PS}_{3}$ (in $d=6-2 \varepsilon$ ), and integrate order by order.

3. Use dimensional recurrence for the resulting integrals to lower the series back to $d=4-2 \varepsilon$.

\footnotetext{
${ }^{1}$ The version of Fuchsia available at github.com/magv/fuchsia.cpp does this automatically.
} 
Overall, this way we get the results as series in $\varepsilon$ up to MZVs of weight 7, with poles up to $1 / \varepsilon^{6}$, and zetas up to weight 6 in the $\varepsilon$-finite part. As an example:

$$
\begin{aligned}
\qquad . & \frac{\mapsto \infty}{\left(q^{2}\right)^{2}}\left[2 \zeta_{2} \frac{1}{\varepsilon}+\left(-13 \zeta_{2}+16 \zeta_{3}\right)+\left(27 \zeta_{2}-104 \zeta_{3}+\frac{156}{5} \zeta_{2}^{2}\right) \varepsilon+\right. \\
& \left.+\left(-18 \zeta_{2}+216 \zeta_{3}-\frac{1014}{5} \zeta_{2}^{2}-90 \zeta_{2} \zeta_{3}+448 \zeta_{5}\right) \varepsilon^{2}+\mathscr{O}\left(\varepsilon^{3}\right)\right] .
\end{aligned}
$$

To cross-check the results we have compared them to the few integrals known from [13], and also by evaluating them numerically using FIESTA [28]. To achieve the latter one needs to find a parametrisation for the cut integrals suitable for sector decomposition; in the case of 3-particle cuts this can be done by combining Feynman parameterization for the loop part, and eq. (3.2) for the phase space.

\section{Cross-check via Cutkosky relations}

Cutkosky relations connect virtual integrals to their cuts; now that we have all cuts of 4-loop propagators available, these can act as the final consistency check. To write them down, for any Feynman diagram $F$ :

$$
F+F^{*}=-\sum_{i} \mathrm{Cut}_{i} F
$$

This simple form is valid for Feynman diagrams, so to use it our integrals must be dressed in Feynman rules; any consistent set would do, and the simplest one comes from a scalar $\phi^{n}$ theory:

$$
\left\langle=\chi=\chi=\cdots=i, \quad \stackrel{\mathrm{p}}{\longrightarrow}=\frac{i}{p^{2}+i 0}, \quad \cdot \stackrel{\mathrm{p}}{\longrightarrow}=2 \pi \delta^{+}\left(p^{2}\right) .\right.
$$

Combining the above, we get one relation for each of the 31 propagator masters:

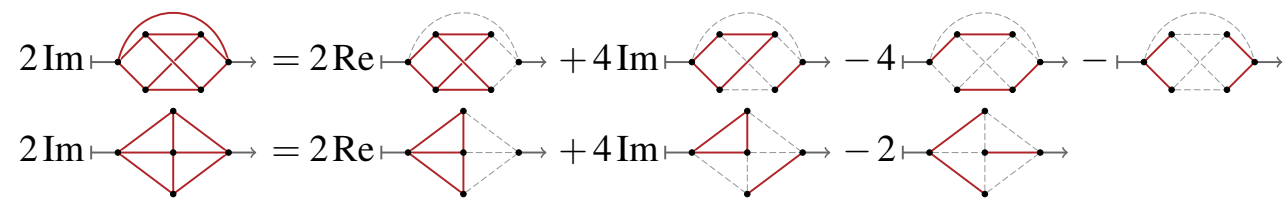

We now have most of the integrals up to weight 12 (with only the 3-particle cuts up to weight 7) inserting these values into these equations gives us our cross-check.

\section{Three-particle cuts via dimensional recurrence relations}

We can additionally use Cutkosky relations to obtain information about one of the cuts from the knowledge of the others, in particular to obtain 3-particle cuts to weight 12 . Two cases need to be considered:

1. The easy case: only one 3-particle cut enters a Cutkosky relation. For example:

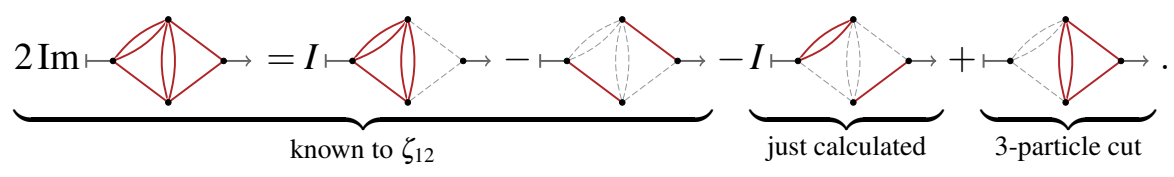


2. The hard case: multiple 3-particle cuts enter a Cutkosky relation. For example:

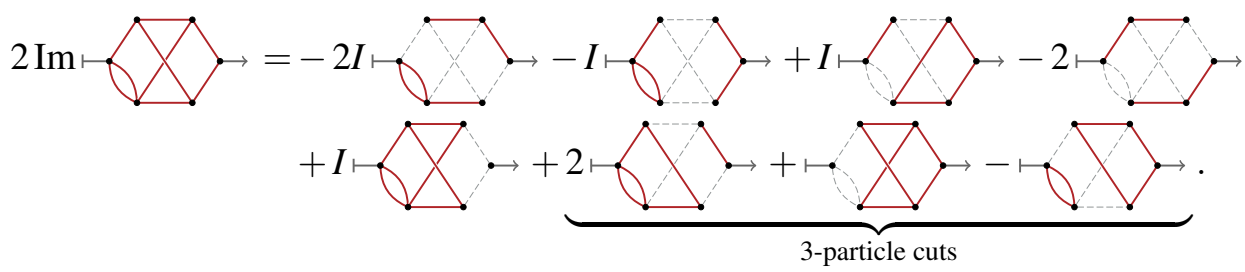

In this case only the sum is constrained. It turns out that this constrain on the sum, together with a few leading terms of the $\varepsilon$-expansion that we have calculated in Section 3 gives us enough information to fix all the constants in the ansatz for $\omega_{i}(d)$ of the DRR solution for 3-particle cut master integrals, and thus solve the DRR for them completely.

This upgrades our knowledge of the 3-particle cuts from weight 7 to weight 12 (and more).

\section{Summary}

We have calculated the full set of master integrals for 3- and 4-particle unitarity cuts of massless 4-loop propagators, thus completing the knowledge of all such cuts. Both direct phase-space integration and the solution of dimensional recurrence relations where used for this. As a byproduct we have also re-calculated the 2-loop $1 \rightarrow 3$ master integrals to transcendental weight 7 (an upgrade from weight-4 result from [23, 24]). Further research includes using these integrals as boundary conditions for the differential master integrals of the same form, and using those for the calculations of NNLO splitting functions.

\section{References}

[1] O. Gituliar, V. Magerya and A. Pikelner, Five-Particle Phase-Space Integrals in QCD, JHEP 06 (2018) $099[1803.09084]$.

[2] V. Magerya and A. Pikelner, Cutting massless four-loop propagators, JHEP 12 (2019) 026 [1910.07522].

[3] S. Moch, B. Ruijl, T. Ueda, J. A. M. Vermaseren and A. Vogt, Four-Loop Non-Singlet Splitting Functions in the Planar Limit and Beyond, JHEP 10 (2017) 041 [1707. 08315].

[4] A. Mitov, S. Moch and A. Vogt, Next-to-Next-to-Leading Order Evolution of Non-Singlet Fragmentation Functions, Phys. Lett. B638 (2006) 61 [hep-ph/ 0604053 ].

[5] S. Moch and A. Vogt, On third-order timelike splitting functions and top-mediated Higgs decay into hadrons, Phys. Lett. B659 (2008) 290 [0 709.3899$].$

[6] A. A. Almasy, S. Moch and A. Vogt, On the Next-to-Next-to-Leading Order Evolution of Flavour-Singlet Fragmentation Functions, Nucl. Phys. B854 (2012) 133 [1107. 2263].

[7] O. Gituliar and S. Moch, Towards three-loop QCD corrections to the time-like splitting functions, Acta Phys. Polon. B46 (2015) 1279 [1505.02901].

[8] O. Gituliar and V. Magerya, Fuchsia: a tool for reducing differential equations for Feynman master integrals to epsilon form, Comput. Phys. Commun. 219 (2017) 329 [1701.04269]. 
[9] O. Gituliar, Master integrals for splitting functions from differential equations in QCD, JHEP 02 (2016) 017 [1512.02045].

[10] G. Heinrich, T. Huber and D. Maître, Master integrals for fermionic contributions to massless three-loop form-factors, Phys. Lett. B662 (2008) 344 [0711.3590].

[11] G. Heinrich, T. Huber, D. A. Kosower and V. A. Smirnov, Nine-Propagator Master Integrals for Massless Three-Loop Form Factors, Phys. Lett. B678 (2009) 359 [0 902 . 3512].

[12] R. N. Lee, A. V. Smirnov and V. A. Smirnov, Analytic Results for Massless Three-Loop Form Factors, JHEP 04 (2010) 020 [1001.2887].

[13] M. Czakon, P. Fiedler, T. Huber, M. Misiak, T. Schutzmeier and M. Steinhauser, The $\left(Q_{7}, Q_{1,2}\right)$ contribution to $\bar{B} \rightarrow X_{s} \gamma$ at $\mathscr{O}\left(\alpha_{\mathrm{s}}^{2}\right)$, JHEP 04 (2015) 168 [1503.01791].

[14] P. A. Baikov and K. G. Chetyrkin, Four Loop Massless Propagators: An Algebraic Evaluation of All Master Integrals, Nucl. Phys. B837 (2010) 186 [1004.1153].

[15] R. N. Lee, A. V. Smirnov and V. A. Smirnov, Master Integrals for Four-Loop Massless Propagators up to Transcendentality Weight Twelve, Nucl. Phys. B856 (2012) 95 [1108 . 0732].

[16] A. Gehrmann-De Ridder, T. Gehrmann and G. Heinrich, Four particle phase space integrals in massless QCD, Nucl.Phys. B682 (2004) 265 [hep-ph/ 0311276$].$

[17] O. V. Tarasov, Connection between Feynman integrals having different values of the space-time dimension, Phys. Rev. D54 (1996) 6479 [hep-th/9606018].

[18] J. Blümlein, D. J. Broadhurst and J. A. M. Vermaseren, The Multiple Zeta Value Data Mine, Comput. Phys. Commun. 181 (2010) 582 [0907.2557].

[19] H. Ferguson, D. Bailey and S. Arno, Analysis of PSLQ, an integer relation finding algorithm, Mathematics of Computation of the American Mathematical Society 68 (1999) 351.

[20] R. N. Lee, Space-time dimensionality D as complex variable: Calculating loop integrals using dimensional recurrence relation and analytical properties with respect to D, Nucl. Phys. $\mathbf{B 8 3 0}$ (2010) 474 [0911.0252].

[21] R. N. Lee and K. T. Mingulov, DREAM, a program for arbitrary-precision computation of dimensional recurrence relations solutions, and its applications, 1712.05173.

[22] R. N. Lee and K. T. Mingulov, Introducing SummerTime: a package for high-precision computation of sums appearing in DRA method, Comput. Phys. Commun. 203 (2016) 255 [1507. 04256].

[23] T. Gehrmann and E. Remiddi, Two loop master integrals for $\gamma^{*} \rightarrow 3$ jets: The Planar topologies, Nucl. Phys. B601 (2001) 248 [hep-ph/ 0008287$].$

[24] T. Gehrmann and E. Remiddi, Two loop master integrals for $\gamma^{*} \rightarrow 3$ jets: The Nonplanar topologies, Nucl. Phys. B601 (2001) 287 [hep-ph/0101124].

[25] A. B. Goncharov, Multiple polylogarithms, cyclotomy and modular complexes, Math. Res. Lett. 5 (1998) 497 [1105.2076].

[26] J. M. Henn, Multiloop integrals in dimensional regularization made simple, Phys. Rev. Lett. 110 (2013) 251601 [1304.1806].

[27] R. N. Lee, Reducing differential equations for multiloop master integrals, JHEP 04 (2015) 108 [1411.0911].

[28] A. V. Smirnov, FIESTA4: Optimized Feynman integral calculations with GPU support, Comput. Phys. Commun. 204 (2016) 189 [1511.03614]. 